\title{
Thrombocytopenia of Unusual Etiology in the Intensive Care Unit
}

\author{
David R Wetzel ${ }^{\mathrm{a}}$, Catherine W Njathi ${ }^{\mathrm{a}}$, Brian J Telesz ${ }^{\mathrm{a}}$, \\ Thomas M Stewart ${ }^{\mathrm{a}}$, Nathan J Smischney ${ }^{\mathrm{b}, \mathrm{c}}$
}

\begin{abstract}
Thrombocytopenia is a relatively common laboratory finding in the intensive care unit (ICU) that may be caused by a variety of triggers. Vancomycin may lead to the production of antibodies that could precipitate a severe drop in platelet count. The objective of the current report was to describe the development of severe thrombocytopenia during the infectious work-up of a critical care patient, where an association between vancomycin and thrombocytopenia was unexpectedly identified. The current report describes the case of a 64-year-old female who presented with dyspnea on exertion and resting hypoxia to an outside facility and was initially treated for a chronic obstructive pulmonary disease exacerbation. She was then transferred to Mayo Clinic for further care. After $72 \mathrm{~h}$, she acutely decompensated requiring increasing oxygen support with a questionable infiltrate on chest imaging and was transferred to the ICU. She was placed on non-invasive ventilation with poor gas exchange and ultimately required intubation. Antibiotics were expanded to include vancomycin. Within $72 \mathrm{~h}$ of antibiotic initiation, her platelet count decreased from $379 \times 10^{9} / \mathrm{L}$ to $7 \times 10^{9} / \mathrm{L}$. After a negative heparin-induced thrombocytopenia panel and serotonin assay, the antibiotics were discontinued and her platelet count subsequently increased. Despite the fact that heparin is a common culprit of drug-induced thrombocytopenia, the patient had no evidence of this association. She received three doses of vancomycin prior to the recognition of the association between this medication and thrombocytopenia. Her platelet count only started to recover once vancomycin was discontinued. In conclusion, vancomycin is commonly administered in the critical care setting and may lead to the development of thrombocytopenia. Vancomycin-induced thrombocytopenia is caused by antibodies that may remain for years after last exposure. Appreciation of the association between vancomycin and thrombocytopenia may ultimately lead to improved patient outcomes.
\end{abstract}

\footnotetext{
Manuscript accepted for publication October 18, 2013

${ }^{\mathrm{a} D e p a r t m e n t ~ o f ~ A n e s t h e s i o l o g y, ~ M a y o ~ C l i n i c, ~ R o c h e s t e r, ~ M N, ~ U S A ~}$

${ }^{\mathrm{b}}$ Critical Care Medicine Division, Department of Anesthesiology, Mayo

Clinic, Rochester, MN, USA

${ }^{c}$ Corresponding author: Nathan Smischney, 200 First Street S.W.,

Rochester, MN 55905, USA. Email: smischney.nathan@mayo.edu

doi: http://dx.doi.org/10.4021/jmc1555w
}

Keywords: Antibiotic; Bleeding; Coagulation; Intensive care unit; Platelet; Thrombocytopenia; Vancomycin

\section{Introduction}

Thrombocytopenia commonly occurs in the intensive care unit (ICU) and may be present in up to $40 \%$ of admissions [1]. The thrombocytopenic patient in the ICU has an increased risk of bleeding and an increased risk of ICU mortality as compared to the non-thrombocytopenic patient $(31 \%$ vs. $15 \%)[1,2]$. The bleeding risk is significantly increased once the platelet nadir is $<100 \times 10^{\%} / \mathrm{L}$ as compared to a nadir of $>100 \times 10^{9} / \mathrm{L}(52 \%$ vs. $21 \%)$ and bleeding complications led to fatality in $23 \%$ of thrombocytopenic patients in the ICU [1]. Common locations of bleeding include gastrointestinal, airways, cutaneous, puncture, and incision sights. Moreover, a low platelet nadir $\left(<150 \times 10^{9} / \mathrm{L}\right)$ or a decrease of platelet count to $<50 \%$ of admission has been demonstrated as an independent predictor of mortality [1].

The cause of thrombocytopenia is frequently multifactorial in origin. However, approximately one-half of thrombocytopenic ICU patients have sepsis as the primary cause and another one-third have infection as the primary cause. Additional causes to consider, in decreasing order of frequency, are liver disease, hypersplenism, disseminated intravascular coagulation or other hematologic disorder, massive transfusion, and medications. Chemotherapeutics, antibiotics, antiplatelet drugs, digoxin, haloperidol, and others have all been associated with thrombocytopenia $[1,3]$.

We present the case of a patient who had the antibiotic vancomycin initiated due to concerns for sepsis and subsequently developed severe thrombocytopenia. Recognition of vancomycin-induced thrombocytopenia led to immediate recovery of platelet count and to the successful outcome of the patient. From this experience, we demonstrate that awareness of vancomycin-induced thrombocytopenia and subsequent withdrawal can lead to rapid recovery of platelet count during the infectious work-up of an ICU patient; thus, vancomycin-induced thrombocytopenia should be recognized early in the course of an infectious work-up in an ICU patient 


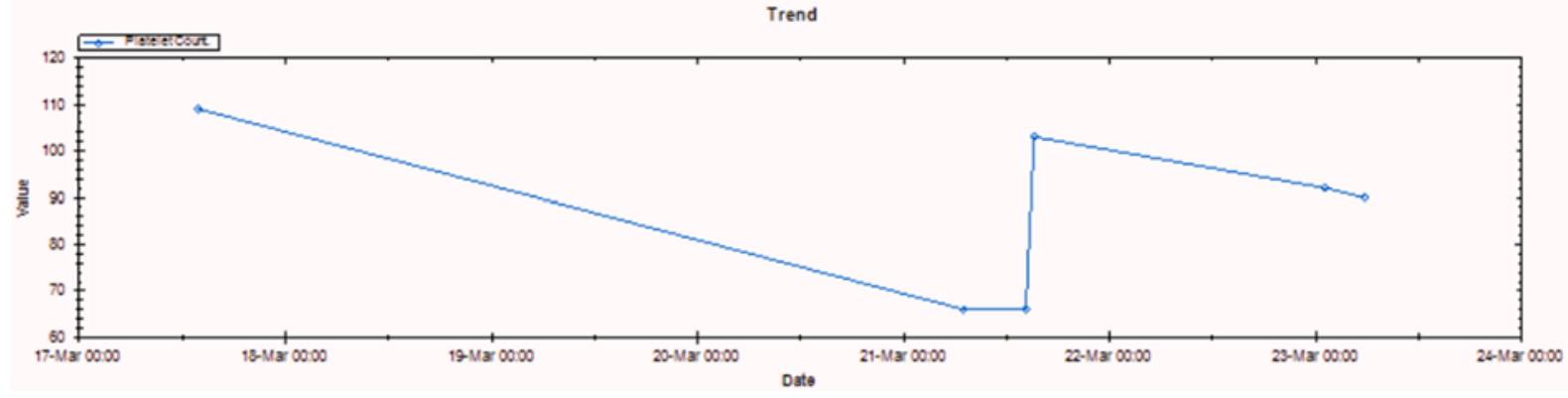

Figure 1. Platelet count trend (March 2009).

who subsequently develops severe thrombocytopenia.

\section{Case Report}

A 64-year-old female with a past medical history significant for chronic obstructive pulmonary disease (COPD) on nocturnal home oxygen therapy, hypertension, obesity, peptic ulcer disease, and chronic lower extremity edema presented to an outside emergency department with complaints of dyspnea on exertion of several days duration. Additionally, she reported upper respiratory tract symptoms of sore throat and cough. Her initial work-up revealed resting hypoxia with oxygen saturations between $85 \%$ and $89 \%$ with mild improvement after nebulizer therapy. She had no chest pain, a normal electrocardiogram, negative troponins, and a normal chest radiograph. She was treated with $750 \mathrm{mg}$ intravenous levofloxacin and transferred to Mayo Clinic Rochester, MN.

On arrival to Mayo Clinic, she was able to communicate in full sentences with notable anxiety. Her initial exam revealed limited air movement with faint expiratory wheezes throughout. Admission labs demonstrated a white blood cell count of $5.5 \times 10^{9} / \mathrm{L}$ with $76 \%$ neutrophils, platelet count of $284 \times 10^{9} / \mathrm{L}$, hemoglobin level of $12.5 \mathrm{~g} / \mathrm{dL}$, arterial blood gas of $\mathrm{pH} 7.36, \mathrm{pCO}_{2} 46, \mathrm{pO}_{2} 166$, and negative influenza swab. Given the initial presentation, she was treated for a COPD exacerbation with oral prednisone $60 \mathrm{mg}$, intravenous levofloxacin $750 \mathrm{mg}$, and scheduled bronchodilator nebulizer treatments.
On hospital day 3 , she was ready for discharge. However, immediately prior to discharge, she acutely decompensated with increased work of breathing and tachypnea requiring increasing oxygen support. A chest radiograph demonstrated a questionable consolidation in the left lower lobe. She was given intravenous furosemide and transferred to the ICU for further management of her hypoxic respiratory failure. Upon arrival to the ICU, she was severely tachypneic with respiratory rates in the high $30 \mathrm{~s}$ and anxious. She was placed on non-invasive ventilation (BiPAP) for support. Initial laboratory work-up in the ICU at 15:13 demonstrated a hemoglobin level of $13.2 \mathrm{~g} / \mathrm{dL}$, white blood cell count of $12.6 \times 10^{9} / \mathrm{L}$, platelet count of $379 \times 10^{9} / \mathrm{L}$, and an arterial blood gas of $\mathrm{pH}$ 7.22, $\mathrm{pCO}_{2} 77, \mathrm{pO}_{2} 223$ after initiation non-invasive ventilation. Her antibiotics were broadened to include vancomycin and cefepime, stress dose steroids were administered, and a computed tomography (CT) scan was performed to assess for possible pulmonary embolism.

After her return from the $\mathrm{CT}$, she deteriorated further necessitating endotracheal intubation. An arterial line and central line were placed without incident. Her CT was reported as showing left lower lobe parenchymal changes consistent with an infectious bronchiolitis and a subsegmental pulmonary embolus in the left lower lobe. The two subsequent complete blood counts at 20:44 and 21:51 were reported as a laboratory error. The third complete blood count at 00:26 demonstrated a platelet count of $7 \times 10^{9} / \mathrm{L}$. The result was rechecked and revealed a platelet count of $9 \times 10^{9} / \mathrm{L}$. She received two units of apheresis platelets with an increase to

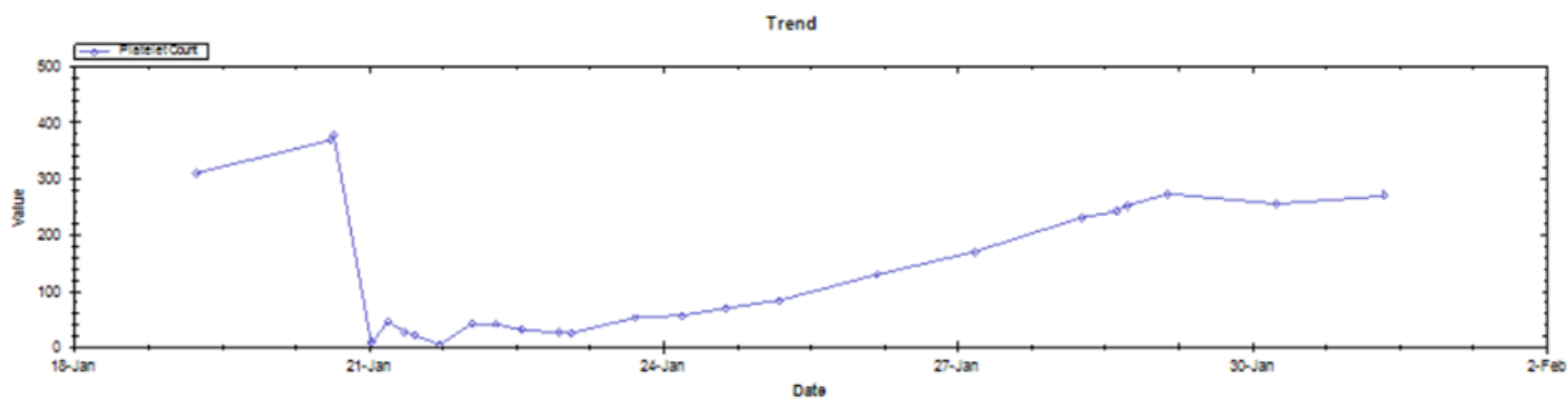

Figure 2. Platelet count trend (January 2013). 
$46 \times 10^{9} / \mathrm{L}$.

The severe thrombocytopenia prompted a coagulopathy work-up. Her hemoglobin remained stable with no laboratory evidence of hemolysis. Her coagulation studies (international normalized ratio and activated partial thromboplastin time) were normal throughout. A thromboelastogram was obtained and showed a slightly prolonged clot kinetic (K) value, decreased alpha angle, and a decrease in maximum amplitude consistent with her thrombocytopenia. A heparininduced thrombocytopenia antibody panel was collected in the setting of recent low molecular weight heparin administration during the hospital course. The value was reported as negative $24 \mathrm{~h}$ later. The remainder of her laboratory assessment was unremarkable, including an electrolyte panel and liver function tests. In addition, her infectious work-up remained negative.

Her platelets declined once again to a nadir of $4 \times 10^{9} / \mathrm{L}$ on ICU day 2 requiring transfusion of two more units of apheresis platelets. To confirm that the thrombocytopenia was not due to heparin-induced thrombocytopenia, a serotonin assay was obtained and reported as negative. One dose of vancomycin was administered at ICU admission and two doses the following day. Antibiotics were discontinued on ICU day 3. Her platelets began to improve, and reached 269 $\times 10^{9} / \mathrm{L}$ on her dismissal 3 days after discharge from the ICU, 8 days after the discontinuation of the vancomycin.

She completed a treatment course for COPD exacerbation consisting of levofloxacin and prednisone and was started on a heparin infusion once her platelet levels stabilized for treatment of her pulmonary embolism. The heparin infusion was transitioned to warfarin upon dismissal. She was discharged without further sequelae.

\section{Discussion}

Thrombocytopenia in the ICU can be the cause of, or result of a multitude of problems. Twenty-five to $38 \%$ of ICU patients will have a platelet count under $100 \times 10^{9} / \mathrm{L}$, while only $2-3 \%$ will have platelet counts below $10 \times 10^{9} / \mathrm{L}$ [4]. While a platelet count below $100 \times 10^{9} / \mathrm{L}$ is considered "thrombocytopenic", clinically significant bleeding often does not occur until the platelet levels are much lower, below $10 \times 10^{9} / \mathrm{L}$ [4]. Conversely, various clinicians have different cut off levels depending on the invasiveness of the procedures, which are often required by ICU patients. The differential diagnosis of thrombocytopenia is broad and ICU patients often have many risk factors, including sepsis, drug therapy (heparin, antibiotics, and so on), disseminated intravascular coagulation, liver and spleen disease, or hematologic disorders [4].

Drug-induced thrombocytopenia is an often overlooked cause in ICU patients due to their multiple comorbidities and ongoing intensive care issues. There is a long list of medications known to cause thrombocytopenia [3]. The estimated incidence is on the order of 10 per million population per year, and often presents $1-3$ weeks after the medication is given with severe thrombocytopenia [5]. The primary therapy is to stop the offending drug and transfuse platelets as dictated by the clinical situation. Other therapies that have been suggested include steroids, immune globulin, and intravenous anti-D antibodies $[5,6]$.

Antibiotics are often implicated in drug-induced thrombocytopenia and are commonly given to ICU patients. Since its introduction, vancomycin has revolutionized the treatment of serious bacterial infections and in particular, infections from Gram-positive cocci. As the number of methicillin-resistant Staphylococcus aureus infections continues to rise, vancomycin has become the cornerstone of antimicrobial therapy in these patients. Unfortunately, there are welldocumented vancomycin-related serious adverse effects such as nephrotoxicity, neutropenia, and an anaphylactoid reaction commonly referred to as Redman's syndrome. In addition, recent data implicate vancomycin in potentially life-threatening thrombocytopenia [6]. It is known to cause an acute, severe thrombocytopenia with an average nadir of $13.6 \times 10^{9} / \mathrm{L}$ a median of 7 days after the initiation of vancomycin (range 3 - 27 days) [6]. The diagnosis is often masked by the numerous confounding issues in these patients such as sepsis, disseminated intravascular coagulation, or a multitude of other medications. The thrombocytopenia is often refractory to platelet transfusions and resolves within days of stopping the vancomycin [6]. Data published by Drygalski et al showed definitively the presence of vancomycin-induced anti-platelet IgM and/or IgG antibodies in 34 patients who had been exposed to drug. Of the 29 patients followed in the study, 10 patients had severe bleeding episodes presumed to be due severe thrombocytopenia. Of the three out of the 10 patients who died, their platelet counts were below $10 \times 10^{9} / \mathrm{L}$. In the 26 patients who survived, the platelet counts returned to baseline once the offending agent was removed [6].

The development and resolution of thrombocytopenia in the present case followed a pattern very similar to that experienced by the patients in the study by Drygalski et al. Interestingly, a review of her medical records indicated our reported incident was not her first episode of severe thrombocytopenia. In 2009, the patient presented to our institution with a lower extremity infection associated with signs and symptoms concerning for sepsis. A broad-spectrum antimicrobial regimen that included vancomycin was initiated. Within $7 \mathrm{~h}$, the patient's platelet count decreased from $300 \times$ $10^{9} / \mathrm{L}$ at admission to $4 \times 10^{9} / \mathrm{L}$ (Fig. 1). At the time, it was unclear whether her profound thrombocytopenia was drugrelated or the result of consumption from disseminated intravascular coagulopathy due to severe sepsis.

A peripheral blood smear indicated myelocytes and nucleated red blood cells indicating a stressed bone marrow, a common finding in severe sepsis. In addition, a serotonin 
release assay was negative for heparin-induced thrombocytopenia. The patient did require six units of packed red blood cells and six units of platelets after she developed significant gastrointestinal bleeding necessitating emergent endoscopy. A review of the patient's medical records indicated that there was concern for possible drug-induced thrombocytopenia, but no specific drug was identified as the possible culprit. At that time, the patient had been exposed to several antimicrobials including vancomycin and piperacillin/tazobactam. These antibiotics were discontinued and patient was started on tigecycline and ciprofloxacin. Eventually (days later and after several platelet transfusions) the patient's platelet count stabilized. As her heparin-induced thrombocytopenia and disseminated intravascular coagulation panels were negative, her thrombocytopenia was attributed to consumption due to severe sepsis.

The patient remained well until our encounter with her in 2013 when she again presented with symptoms concerning for possible sepsis. As in her previous episode in 2009, she developed severe thrombocytopenia that was refractory to platelet infusions. A comparison of the course of her thrombocytopenia during the two documented episodes shows a very similar pattern on both occasions that is also comparable to the pattern seen by Drygalski et al. Once again, serotonin release assay was negative for heparin-induced thrombocytopenia. After her second dose of vancomycin, we became highly suspicious of the drug after her thrombocytopenia worsened. The vancomycin was discontinued following the third dose after a clear pattern of worsening thrombocytopenia within $4 \mathrm{~h}$ of each dose was established. Her platelet count subsequently stabilized and returned to baseline by discharge (Fig. 2). Of note, our patient actually required heparin for a pulmonary embolism well after her thrombocytopenia had resolved. She tolerated the treatment well without recurrence of her thrombocytopenia, further confirming that her thrombocytopenia was not due heparininduced thrombocytopenia.

\section{Conclusions}

Thrombocytopenia is relatively common in the ICU and may be caused by a wide variety of triggers or etiologies. After exclusion of common causes, drug-induced thrombocytopenia should be recognized as a possible cause. Vancomycin is commonly administered in this setting and may lead to the development of thrombocytopenia. Vancomycin-induced thrombocytopenia is caused by antibodies that may remain for years after last exposure. Appreciation of vancomycininduced thrombocytopenia may ultimately lead to improved patient outcomes.

\section{Funding}

No grants received for this work.

\section{Conflict of Interest}

All authors declare no conflicts of interest.

\section{Disclosure}

A portion of this work in the form of an abstract has been submitted to the Society of Critical Care Medicine 2014.

\section{References}

1. Vanderschueren S, De Weerdt A, Malbrain M, Vankersschaever D, Frans E, Wilmer A, Bobbaers H. Thrombocytopenia and prognosis in intensive care. Crit Care Med. 2000;28(6):1871-1876.

2. Strauss R, Wehler M, Mehler K, Kreutzer D, Koebnick C, Hahn EG. Thrombocytopenia in patients in the medical intensive care unit: bleeding prevalence, transfusion requirements, and outcome. Crit Care Med. 2002;30(8):1765-1771.

3. Arnold DM, Nazi I, Warkentin TE, Smith JW, Toltl LJ, George JN, Kelton JG. Approach to the diagnosis and management of drug-induced immune thrombocytopenia. Transfus Med Rev. 2013;27(3):137-145.

4. Irwin RS, Rippe JM. Intensive Care Medicine. Seventh Edition. Philadelphia. Lippincott, Williams \& Wilkins, 2012.

5. Aster RH, Bougie DW. Drug-induced immune thrombocytopenia. N Engl J Med. 2007;357(6):580-587.

6. Von Drygalski A, Curtis BR, Bougie DW, McFarland JG, Ahl S, Limbu I, Baker KR, et al. Vancomycininduced immune thrombocytopenia. N Engl J Med. 2007;356(9):904-910. 